hardnesse ; whereupon I desired him to cut the Orifice wider: by which meanes we presently perceived a carnouse substance, as it seemed to us wreathed together in foldes like a worme or Serpent: at which we both much wondred, and I intreated him to seperate it from the heart, which he did, and we carryed it from the body to the window, and there layed it out, in those just dimensions which are here expressed in the figure.

"The body was white of the very colour of the whitest slin of mans body: but the skin was bright and shining, as if it had beene varnished over; the head all bloody, and so like the head of a Serpent, that the Lady Herris then shivered to see it, and since hath often spoken it, that she was inwardly troubled at it, because the head of it was so truely like the head of a Snake.

"The thighes and branches were of flesh colour, as were also all these fibraes, strings, nerves, or whatsoever else they were.

"After much contemplation and conjectures what strange thing that part of the heart had brought forth unto us, I resolved to try the certainty, and to make full exploration, both for mine owne experience and satisfaction, as also to give true testimony to others that should heare of it: And thereupon searched all parts of it, to find whether it were a pituitose and bloody Collection, or the like: Or a true organicall body, and Conception: I first searched the head and found it of a thicke substance, bloody and glandulous about the necke, somewhat broken (as I conceived) by a sudden or violent separation of it from the heart, which yet seemed to me to come from it easily enough.

"The body I searched likewise with a bodkin betweene the Legs or Thighs, and I found it perforate, or hollow, and a solid body, to the very length of a silver bodkin, as is here described: At which the Spectators wondered. And as not crediting me, some of them tooke the bodkin after me, made triall them selves, and remained satisfied, that there was a gut, Veine or Artery, or some such Analogicall thing that was to serve that Monster for uses naturall: Amongst whom the Lady Herris and the Surgian made tryall after me with their own hands, and have given their hands that this Relation is true. This Lady dwelleth at the signe of the Sugar loafe in S. Iames street in the Convent Garden."

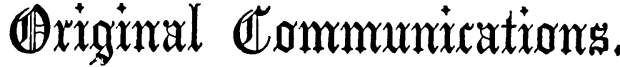

\section{TREATMENT OF TETANUS BY ACONITE}

By Leonard W. SedGwick, M.D., Boroughbridge. Aconite having been recently recommended by Mr. Page and Mr. Campbell de Morgan as worthy of fair trial in tetanus, and all old established methods of treatment having failed in affording any hope of cure in this formidable disease, I thought myself justified in subjecting a case which came under my care last May to the full influence of the drug. Before offering any remarks, I will give a condensed history of the case from detailed notes taken at the time.

Case. A farmer's labourer, aged 30, strong and muscular, healthy and temperate, in jumping off a cart with a dung.fork in his hand, stuck it into bis left thigh about three inches above the knee-joint on the inner and anterior surface. For some days the knee was stiff, and he was unable to work. Ten days afterwards, being much better, he began chopping sticks. About noon he got wet; and, whilst at his dinner, he was seized with a sharp pain between his shoulders; at the same time he thought he could not open his mouth as well as usual. On the eleventh day, he was unable to work from acute pain in the back and jaws.

On the twelfth day from the accident and the third of the tetanus, I saw him. He was then lying on his back, perspiring intensely, with an anxious painful expression of countenance. His jaws were nearly closed; the muscles of the back were very rigid; the loins were almost always some distance off the bed; the arms were not much affected; the leg were stiff; the abdominal muscles hard. Pulse 95, not very full. His tongue was moderately clean. The bowels wer regular. He had no sleep. The urine was natural. I ordered him beef-tea and six ounces of brandy in the day; and five minims of Fleming's tincture of aconite in water every four hours. I laid open the wound, and removed a considerable piece of woollen cloth, which had been driven in from his trousers by the fork. To save repetition, I may here state that the wound healed steadily.

Fourth day. He was much the same.

Fifth day. The spasms were not so constant, but more violent. No aconitism had appeared. Seven minims of the tincture of aconite were given every four hours.

Sixth day. He was worse. When the spasms were relaxed, which was only for a very few minutes, the pulse was 68 ; during the spasms, it rapidly rose to 120 , and became smaller. Opisthotonos was extreme; the jaws were clenched. Ten minims of the tincture were given every four hours; and he was ordered to have ten ounces of brandy daily.

Seventh day. Tingling in the hands and feet and slight giddiness having come on, the spasms had been much less severe. The pulse was weaker, and he had great sleeplessness and restlessness. The aconite was omitted; and twenty minims of chlorodyne were given in an ounce of water every four hours.

Eighth day. He continued easier, and slept well. The pulse was stronger. The spasms were not so frequent. $\mathrm{H}$ complained of much pain from flatulence. Twenty minims of tincture of sumbul were given with the chlorodyne. I may remark, that I have seen more benefit from sumbul in flatulence than from any other drug.

Ninth day. He took more beef-tea, etc., and was improving. The flatulence was diminished.

Tenth day. Immediately after being startled by a loud noise, he had a violent spasm, lasting some time. It recurred at intervals with great violence. He was ordered to have three minims of tincture of aconite and ten of chlorodyne in an ounce of water every four hours.

Eleventh day. He was much the same. The dose of tincture of aconite was increased to four minims.

Notbing of importance occurred until the seventeenth day. $\mathrm{He}$ continued the mixture, and the cramp decreased. The bowels having been confined several days, he had a turpentine enema, which greatly relieved him, and was repeated every other day. On the seventeenth day, some tingling came on, and continued until the nineteenth, though the aconite was reduced to a minim and a half every four hours. On that day, the aconite was suspended. The next day there was more cramp. The aconite was resumed for a week longer, and he gradually recovered. In less than three months he was at work again. The muscles were some time in regaining their extensibility after the tetanic spasms had ceased.

REITAKS. The subjects for consideration are, the severity of the attack; the action of the aconite; the effect of the chlo. rodyne; the chances of spontaneous recovery; and the value of the case as a guide to future treatment.

It was unquestionably a most acute attack. A punctured wound, containing an irritating foreign body, prevents a man from working for ten days. He gets wet; and immediately tetanus sets in, and rapidly worsens. Every muscle of the body is racked with cramp; more than half his time he rests on his head and his heels; every step across the floor, every noise in the house, agitates him with this torturing spasm ; and from this agony he is free for only two or three minutes at a time. To those who saw him, it was truly a most severe case.

If I have related honestly and faithfully what I most certainly saw, there will be little reason for any detailed argument as to the action of the aconite. When the symptoms of aco nitism came on, then, and not till then, did the symptoms begin to abate. Twice, when the aconite was suspended, once to try the effect, once because the tingling and giddiness were becoming extreme, did the spasms return, to abate again on the resumption of the drug. This contratetanic action of the aconite is rendered more probable by the large doses which were originally borne, $\mathbf{a}$ part of the remedy seeming to be neutralised, as it were, by the disease, and only the superfluity able to produce its poisonous effect. This view will explain why smaller doses produced greater effects towards the end of the case, there being then less disease to combat.

Perhaps some justification is necessary of the administration of the chlorodyne. Perhaps it may be objected, that I marred the decision of the result by its use. I do not think so. I have often seen the good effects of chlorodyne in producing quiet without depression. Here was a man under the influ. ence of aconite-sleepless, intensely and emphatically restless. My object was to cure him, not to conduct an experiment only. I saw reason to believe that chlorodyno would relieve the disagreeable symptoms probably produced by the aconite, 
and it did so. But there was no repression of the spasms as a result of its use; for twice it was given alone, and each time the spasms returned, to be reduved again by the aconite. Useful, then, in relieving a disagreeable accident, it had no power to stand against the tetanus.

If $I$ have succeeded in demonstrating the acute nature of the attack, the extremity of the symptoms, and the contraspasmodic action of the aconite, there can be little need to say anything of the chances of spontaneous recovery. He might have beaten the disease; but, from all we know of tetanus, we may be pretty sure that it would have gained the day.

What, then, is the value of the case? Does it contain hope for the future, or is its successful termination merely a for turiate accident? I cannot but think that it is full of hope, more especially when taken in connexion with previously reported cases. Of course it is not conclusive; it cannot be. But it points very decidedly to a certain path, by following which there is more hope of arriving at the wished for goal than by the old well trodden tracks. Aconite has never had that thorough testing that opium, for instance, bas. So far, it seems to me most useful : further experience will determine its true value. But little persuasion should be required to induce a fair and extended trial of its powers, not by one or two but by many; for, in such matters as this, "in a multitude of counsellors there is safety". Nor need we restrict our notice to aconite alone; it is only one of a class of medicines which has yet had little attention paid to it-all powerful, even vio lent, in their aetion, and many, I am convinced, containing " a soul of good" within them, which as yet we have not "observingly distilled out".

\section{ANOMALOUS CASE IN A CHILD.}

\section{By D. W. Sargent, Esq., Camberwell.}

The following case, which lately occurred in my practice, appears so anomalous that I am induced to reeord it. I am unable to recognise the symptoms, in the course which they followed, as belonging to any known disease; and the post mortem examination did not reveal any condition sufficient to account for death.

CASE. L. D., a fine healthy boy, of sanguine temperament, aged 3 years and 9 months, was quite well on December 29th, and was naturally relieved in his bowels three times on that day.

Dec. 30th. He was very sick and restless throughout the day: the ingesta only, with mucus, were vomited. The pulse was 84 , feeble; the skin cool. He was very thirsty; the bowels were inactive.

Dec. 31st. He had nausea, but no vomiting; nor was there purging. There was no febrile heat. He was very restless and thirsty. The urine was natural, and passed freely. The pulse was 84 ; the skin cool; and the head clear.

Jan. 1st, 1860. He vomited very frequently, and was very thirsty. The urine was plentiful and natural. The skin was colder. He was perfectly conscious, and had no convulsions. The breathing, which had been panting, became more so, and there was more play of the nostrils; but there were no bronchitic nor pneumonic sounds. The pulse was 96 , thready. $\mathrm{He}$ dozed occasionally for a few minutes, apparently from prostration. The pupils were natural or dilated, never contracted. The bowels were costive.

Jan. 2nd. All the symptoms continued, with more restless tossing and panting, and colder skin. The vomiting ceased in the evening. The urine was passed freely : there was no action of the bowels. He had no perspiration, cramps, or convul. sions : he dozed frequently, but was perfectly conscious.

Jan. 3rd. After taking castor oil, he had a motion, natural in character, early in the morning. The vomiting did not return. He said once or twice that he was "better now"; but the coldness, panting, and sinking increased hourly.

Jan. 4th. Diffused warmth appeared over the surface in the morning ; just after which he quietly breathed his last, in perfect consciousness.

The only indication of pain throughout the case was an occasional pointing to the throat just above the sternum ; but the throat was perfectly free from disease. No eruption at any time appeared on the skin.

The treatment consisted at first in small doses of dilute nitric acid, to allay vomiting; and two calomel purges were given. Afterwards, bicarbonate of potash and aromatic spirits of ammonia were used; then solution of carbonate of am- monis; and subsequently, dilute sulphuric acid. Through out the case, milk and wine, and lastly brandy, were given, according to circumstances.

Post Mortem Examination. The brain was free from lesion throughout. There was no inflammation, nor extravas. ation of blood, serum, or pus, on the surface of the cerebrum or in the ventricles; neither was there any on the crura, the pons Varolii, or medulla oblongata, or beneath the pia mater, in the cavity of the arachnoid, between the arachnoid and dura mater, or between the dura mater and skull. All these parts were free from opacity or thickening. There was some vas cular injection of the membranes at the base of the cerebellum but it was removable by washing. No trace of disease could be found in the lungs and heart. The left auricle contained a soft natural coagulum; the left ventricle was empty. The right auricle and ventricle contained slightly coagulated blood. There was no engorgement of the pulmonary vessels. The stomach was half full of a straw-coloured watery fluid, which was not tested. The organ appeared healthy: the mucous coat was not injected. The liver was natural in size and external outline, and presented no morbid appearance. The intestinal canal was healthily distended, and was free from injection or inflammation. The bladder was quite empty and collapsed.

\section{TWO CASES OF FALLS FROM HEIGHTS FOL- LOWED BY VERY SLIGHT INJURY.}

By George MaLletr, Esq., Bolton-le-Moors.

Is a recent number of the JouRnad, Dr. Fleming writes that we ought to record only good cases; meaning such as present something new and of living interest in diagnosis or treatment, or illustrative of some practical rule already established. There appears to me to be so much of truth and wisdom in these remarks, that they ought to command universal assent; at any rate, they meet with my cordial approbation. Nevertheless, I am afraid I am going to act in opposition to them, by giving a brief relation of two cases, that would scarcely be ranked amongst those that ought to be published, if we construe the above rule very rigidly, as they do not indicate anything novel either in treatment or diagnosis.

I send this paper for publication, believing that similar cases to these, therein related, cannot often occur, without suspending on their behalf one of the ordinary laws of naturethat of gravitation.

The two following cases are those of men falling from such heights as would, under most circumstances, be followed by instant death, but they escaped; and therefore I think they are sufficiently interesting to entitle them to be placed upon record.

CASE I occurred about sixty years ago; and about twentyfive or twenty-six years since, it came to my knowledge under the following circumstances.

At the period last named, I was requested to visit a poor man, who had been suffering severely for four days. I found him in great agony from retention of urine, none of which had passed from the bladder for four days. Many ineffectual attempts had been made for his relief by an irregular practitioner, but, unfortunately, he had been unable to introduce the catheter. The patient was about 70 years old, and presented the following symptoms. The skin was hot; the face red, and much emaciated; the pulse was rapid and very feeble; the mouth and tongue hot, parched, and covered by a thick coating of dark fur-in fact, presenting such an appearance as would be expected from so long a retention of urine. With great difficulty, a large catheter was introduced, and from five to six pints of urine were drawn off: the first was very ammoniacal, and the latter portion semipurulent and bloody. The catheter was introduced twice a day for three days, when the man died.

The day before his death, he was very cheerful; and, in a jocular manner, said, " that in his younger days he had met with a bad fall, but he feared he had then suffered a worse one, as he had fallen into the hands of the doctor." He explained himself by stating, that when placing some of the masonry upon the highest part of the tower of a church, called St. George's, in this town, his foot slipped, and he was precipitated from the top to the bottom, the distance being at least one hundred and twenty feet; and that the fall was uninterrupted by any intervening scaffolding, so as to diminish the impetus of the descent. He added, that he was so little in- 